\title{
Stabilization of Two Electricity Generators
}

\author{
Dany Ivan Martinez, José de Jesús Rubio $\mathbb{D}$, Arturo Aguilar, Jaime Pacheco $\mathbb{D}$, \\ Guadalupe Juliana Gutierrez, Victor Garcia, Tomas Miguel Vargas, Genaro Ochoa $\mathbb{D}$, \\ David Ricardo Cruz $\mathbb{B}$, and Cesar Felipe Juarez
}

\begin{abstract}
Sección de Estudios de Posgrado e Investigación, ESIME Azcapotzalco, Instituto Politécnico Nacional, Av. De Las Granjas No. 682, Col. Santa Catarina, Ciudad de México 02250, Mexico
\end{abstract}

Correspondence should be addressed to José de Jesús Rubio; rubio.josedejesus@gmail.com

Received 2 July 2020; Revised 20 November 2020; Accepted 26 November 2020; Published 10 December 2020

Academic Editor: Hamid Reza Karimi

Copyright (c) 2020 Dany Ivan Martinez et al. This is an open access article distributed under the Creative Commons Attribution License, which permits unrestricted use, distribution, and reproduction in any medium, provided the original work is properly cited.

In this research, a sliding mode regulator with sine mapping is suggested for the stabilization of electricity generators being affected by magnet interaction nonlinearities and generator nonlinearities. To reach this goal, our suggested regulator has the following contributions: (a) it starts from the sliding mode regulator with the modifications that the saturation mapping is used to reach a smoother performance instead of the signum mapping, and the sine mapping is applied to reach an upper bound in the proportional gain error, (b) it is used to reach some chosen constant behaviors for the angle position, angle speed, and current in the electricity generators, and (c) its stabilization is ensured based on the Lyapunov approach. We show the simulation of the suggested regulator in two electricity generators.

\section{Introduction}

The term alternative energy is utilized in the electricity generation from the environment by the utilization of renewable fuels, evading the necessity of a no renewable fuel. Some of the most relevant alternative energies can be classified in electricity generators based on wind turbines, magnets, or solar panels. The electricity generator of this research uses the interactions between static and dynamic magnets for the electricity generation $[1,2]$. The motivation of this research is the stabilization of electricity generators. A regulator is one technique for the stabilization of electricity generators which let us to reach some constant behaviors for the angle position, angle speed, and current [3-8].

There are many kinds of nonlinearities which impede to reach the stabilization of electricity generators, and some examples of these nonlinearities are the arbitrary switching
[9-13], the time-delays [14-18], the impulse perturbations $[19,20]$, or the unknown nonlinearities [21-24]. The major issue is that in most of the cases, the mentioned nonlinearities are unknown. The challenge of this research is the stabilization of electricity generators being affected by unknown nonlinearities.

The magnet interaction nonlinearities and generator nonlinearities are two unknown nonlinearities of the electricity generators which can severely affect their performance [21-24]. Hence, the research about regulators for the stabilization of electricity generators where the magnet interaction nonlinearities and generator nonlinearities are unknown, which is of interest, and two alternatives are the proportional derivation regulator of [3-5] and the sliding mode regulator of [6-8].

In this research, a sliding mode regulator with sine mapping is suggested for the stabilization of electricity 
generators being affected by magnet interaction nonlinearities and generator nonlinearities. To reach this goal, our suggested regulator has the following contributions:

(a) The sliding model regulator starts from the proportional derivation regulator with one additional term of the signum mapping used to reach the stabilization. The sliding mode regulator with sine mapping starts from the sliding mode regulator with the modifications that the saturation mapping is used to reach a smoother performance instead of the signum mapping, and the sine mapping is applied to reach an upper bound in the proportional gain error.

(b) Since the sliding mode technique is used in the suggested regulator instead of the magnet interaction nonlinearities and generator nonlinearities, the knowledge of these nonlinearities is not required in the electricity generators. Our suggested regulator is also used to reach some chosen constant behaviors for the angle position, angle speed, and current.

(c) We ensure the stabilization of the regulator error in our suggested regulator based on the Lyapunov approach. This research is focused on the asymptotic stabilization.
We organize the paper as follows: in Section 2, we present the mathematical model of electricity generators. In Section 3, we present the sliding mode regulator with sine mapping for the stabilization of electricity generator. In Section 4, we simulate the suggested regulator for the stabilization of two electricity generators. In Section 5 we present the conclusions and future research.

\section{Mathematical Model and Regulators for the Electricity Generators}

In Appendix A of this research, the descriptions of the electricity generator symbols are shown in Table 1 and the parameter values for mathematical models are shown in Table 2. The descriptions of the mathematical model symbols are shown in Table 3, and the descriptions of the regulator symbols are shown in Table 4.

In this section, we address some concepts such as the mathematical model and regulators for the electricity generators.

We consider the electricity generator with two static magnets and two dynamic magnets of Figure 1 via the utilization of two static magnets and two dynamic magnets.

We express the electricity generator with two static magnets and two dynamic magnets as $[1,2]$

$$
\begin{aligned}
\dot{z}_{1}= & z_{2}, \\
\dot{z}_{2}= & \left(\frac{1}{m_{e}\left(1 / 24 a^{2}+1 / 24 c^{2}+r_{g}^{2}\right)+m_{i}\left(r_{i}^{2}+2 r_{g}^{2}\right)}\right) \\
& \cdot\left(v-\frac{\pi B^{2} r_{i}^{2} r_{g}^{2} \sin z_{1}}{\mu\left[h^{2}+2 r_{g}^{2}\left(1-\cos z_{1}\right)\right]^{0.5}}+\frac{\pi B^{2} r_{i}^{2} r_{g}^{2} \sin z_{1}}{\mu\left[h^{2}+2 r_{g}^{2}\left(1+\cos z_{1}\right)\right]^{0.5}}-b z_{2}\right), \\
\dot{z}_{3}= & -\frac{\left(R+R_{e}\right)}{L} z_{3}+\frac{K_{m}}{L} z_{2} .
\end{aligned}
$$

Since all magnets are equal, $B_{11}=B_{12}=B$. 
TABLE 1: Description of the electricity generator symbols.

\begin{tabular}{|c|c|}
\hline Symbol & Description \\
\hline$m_{e}$ & The mass of the link 1 \\
\hline$a$ & The width of the link 1 \\
\hline$c$ & The length of the link 1 \\
\hline$m_{i}$ & The mass of the dynamic magnet \\
\hline$r_{i}$ & The radius of the surface in the magnet \\
\hline$m$ & The mass \\
\hline$r_{g}$ & The radius of the body rotation \\
\hline$B_{11}^{y}$ & The magnet flux density produced by dynamic magnet 1 and static magnet 1 \\
\hline$B_{12}$ & The magnet flux density produced by dynamic magnet 1 and static magnet 2 \\
\hline$B_{21}$ & The magnet flux density produced by dynamic magnet 2 and static magnet 1 \\
\hline$B_{22}$ & The magnet flux density produced by dynamic magnet 2 and static magnet 2 \\
\hline$\mu$ & The magnet permeability constant in magnets \\
\hline$h$ & The distance of a dynamic magnet and a static magnet on the axis $z$ \\
\hline$K_{m}$ & The electromotive force constant \\
\hline$R$ & The resistance \\
\hline$i$ & The current \\
\hline$L$ & The inductance \\
\hline$R_{e}$ & The load resistance \\
\hline
\end{tabular}

We consider the electricity generator with four static magnets and four dynamic magnets of Figure 1 via the utilization of four static magnets and four dynamic magnets.
We express the electricity generator with four static magnets and four dynamic magnets as $[1,2]$

$$
\begin{aligned}
\dot{z}_{1}= & z_{2}, \\
\dot{z}_{2}= & \left(\frac{1}{m_{e}\left(1 / 24 a^{2}+1 / 24 c^{2}+r_{g}^{2}\right)+m_{i}\left(r_{i}^{2}+2 r_{g}^{2}\right)}\right)\left(v-\frac{\pi B^{2} r_{i}^{2} r_{g}^{2} \sin z_{1}}{\mu\left[h^{2}+2 r_{g}^{2}\left(1-\cos z_{1}\right)\right]^{0.5}}\right. \\
& -\frac{\pi B^{2} r_{i}^{2} r_{g}^{2} \cos z_{1}}{\mu\left[h^{2}+2 r_{g}^{2}\left(1+\sin z_{1}\right)\right]^{0.5}}+\frac{\pi B^{2} r_{i}^{2} r_{g}^{2} \sin z_{1}}{\mu\left[h^{2}+2 r_{g}^{2}\left(1+\cos z_{1}\right)\right]^{0.5}}+\frac{\pi B^{2} r_{i}^{2} r_{g}^{2} \cos z_{1}}{\mu\left[h^{2}+2 r_{g}^{2}\left(1-\sin z_{1}\right)\right]^{0.5}}-\frac{\pi B^{2} r_{i}^{2} r_{g}^{2} \sin \left(z_{1}-\pi / 4\right)}{\mu\left[h^{2}+2 r_{g}^{2}\left(1-\cos \left(z_{1}-\pi / 4\right)\right)\right]^{0.5}} \\
& -\frac{\pi B^{2} r_{i}^{2} r_{g}^{2} \cos \left(z_{1}-\pi / 4\right)}{\mu\left[h^{2}+2 r_{g}^{2}\left(1+\sin \left(z_{1}-\pi / 4\right)\right)\right]^{0.5}}+\frac{\pi B^{2} r_{i}^{2} r_{g}^{2} \sin \left(z_{1}-\pi / 4\right)}{\mu\left[h^{2}+2 r_{g}^{2}\left(1+\cos \left(z_{1}-\pi / 4\right)\right)\right]^{0.5}}+\frac{\pi B^{2} r_{i}^{2} r_{g}^{2} \cos \left(z_{1}-\pi / 4\right)}{\left.\mu\left[h^{2}+2 r_{g}^{2}\left(1-\sin \left(z_{1}-\pi / 4\right)\right)\right]^{0.5}-b z_{2}\right),} \\
\dot{z}_{3}= & -\frac{\left(R+R_{e}\right)}{L} z_{3}+\frac{K_{m}}{L} z_{2} .
\end{aligned}
$$

Since all magnets are equal, $B_{11}=B_{12}=B_{21}=B_{22}=B$.

From the electricity generator with two static magnets and two dynamic magnets of equation (1) and the electricity generator with four static magnets and four dynamic magnets of equation (2), we express the mathematical model for the electricity generators as 
TABle 2: Parameters of electricity generators.

\begin{tabular}{lccc}
\hline Parameter & Value & Parameter & Value \\
\hline$m_{i}$ & $9.2 \times 10^{-2} \mathrm{~kg}$ & $R_{e}$ & $30 \Omega$ \\
$\mu$ & $9.42 \times 10^{-5} \mathrm{Hm}^{-1}$ & $r_{g}$ & $7.5 \times 10^{-2} \mathrm{~m}$ \\
$B$ & $8.4 \times 10^{-1} \mathrm{~T}$ & $r_{i}$ & $2.9 \times 10^{-2} \mathrm{~m}$ \\
$L$ & $6.03 \times 10^{-1} \mathrm{H}$ & $a$ & $1.5 \times 10^{-1} \mathrm{~m}$ \\
$K_{m}$ & $45 \times 10^{-2} \mathrm{Vsrad}^{-1}$ & $c$ & $1.5 \times 10^{-2} \mathrm{~m}$ \\
$b$ & $1 \times 10^{-1} \mathrm{kgm}^{2} \mathrm{rads}^{-1}$ & $n_{r}$ & 0.5 \\
$m_{e}$ & $5 \times 10^{-3} \mathrm{~kg}^{-}$ & $n_{l}$ & 0.5 \\
$h$ & $4 \times 10^{-2} \mathrm{~m}$ & $w_{r}$ & 0.5 \\
$R$ & $6.96 \Omega$ & $w_{l}$ & -0.5 \\
\hline
\end{tabular}

TABLe 3: Description of the mathematical model symbols.

\begin{tabular}{lc}
\hline Symbol & Description \\
\hline$z_{1} \in \mathfrak{R}$ & The angle position \\
$z_{2} \in \mathfrak{R}$ & The angle speed \\
$z_{3} \in \mathfrak{R}$ & The current \\
$v \in \mathfrak{R}$ & $\begin{array}{c}\text { The fictitious input of torque produced by the magnets interaction } \\
O\left(z_{1}\right) \in \mathfrak{R}\end{array}$ \\
$z_{1}, z_{2}, z_{3}$ & The magnets interaction nonlinearities \\
$Q, J, X, Y$ & The states \\
$u$ & Scalar constants \\
$n_{r}, n_{l}, w_{r}, w_{l}$ & The input of the generator nonlinearities \\
$P(u)$ & Constant terms for the generator nonlinearities \\
\hline
\end{tabular}

TABLE 4: Description of the regulators symbols.

\begin{tabular}{lc}
\hline Symbol & Description \\
\hline$\widetilde{z}_{1}=z_{1}-z_{1}^{d} \in \mathfrak{R}$ & The position regulator error \\
$z_{1}^{d} \in \mathfrak{R}$ & The constant reference position \\
$\widetilde{z}_{2}=z_{2} \in \mathfrak{R}$ & The speed regulator error \\
$K_{1} \in \mathfrak{R}$ & The proportional gain \\
$K_{2} \in \mathfrak{R}$ & The derivation gain \\
$\operatorname{sign}(\cdot)$ & The signum mapping \\
$\operatorname{sat}(\cdot)$ & The saturation mapping \\
$\sin (\cdot)$ & The sine mapping \\
$\cos (\cdot)$ & The cosine mapping \\
$K$ & The sliding mode gain \\
$\widetilde{z}_{1}^{2}=\left(z_{1}-z_{1}^{d}\right)^{2}$ & The position regulator error \\
$\widetilde{z}_{2}^{2}=z_{2}{ }^{2}$ & The speed regulator error \\
$\widetilde{z}_{3}^{2}=z_{3}{ }^{2}$ & The current regulator error \\
\hline
\end{tabular}

$$
\begin{aligned}
\dot{z}_{1} & =z_{2} \\
Q \dot{z}_{2}+J z_{2}+O\left(z_{1}\right) & =v \\
\dot{z}_{3}+X z_{3}+Y z_{1} & =0
\end{aligned}
$$

The fictitious input $v$ is affected by the generator nonlinearities, it is like a chattering movement presented in the electricity generator. We express the fictitious input $v$ as [6]

$$
v= \begin{cases}n_{r}\left(u-w_{r}\right), & u \geq w_{r} \\ 0, & w_{l}<u<w_{r} \\ n_{l}\left(u-w_{l}\right), & u \leq w_{l}\end{cases}
$$

We see that the model of equation (1) can be expressed as the model of equations (3) and (4) with

$$
\begin{aligned}
Q & =m_{e}\left(\frac{1}{24} a^{2}+\frac{1}{24} c^{2}+r_{g}^{2}\right)+m_{i}\left(r_{i}^{2}+2 r_{g}^{2}\right), \\
J & =b \\
X & =\frac{\left(R+R_{e}\right)}{L}, \\
Y= & -\frac{K_{m}}{L}, \\
O\left(z_{1}\right)= & \frac{\pi B^{2} r_{i}^{2} r_{g}^{2} \sin z_{1}}{\mu\left[h^{2}+2 r_{g}^{2}\left(1-\cos z_{1}\right)\right]^{0.5}} \\
& -\frac{\pi B^{2} r_{i}^{2} r_{g}^{2} \sin z_{1}}{\mu\left[h^{2}+2 r_{g}^{2}\left(1+\cos z_{1}\right)\right]^{0.5}} .
\end{aligned}
$$




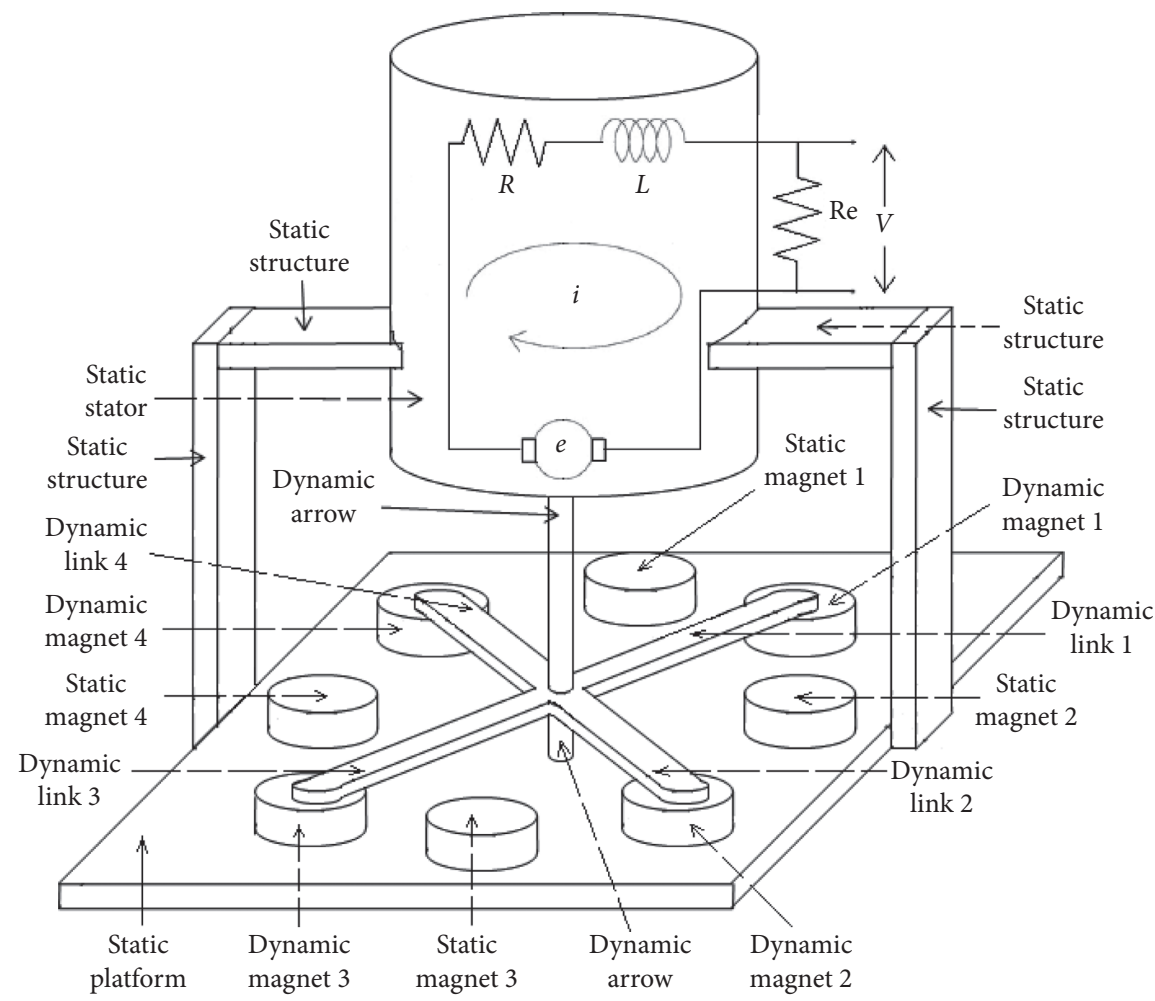

FIGURE 1: Electricity generator with static magnets and dynamic magnets.

We see that the model of equation (2) can be expressed as the model of equations (3) and (4) with

$$
\begin{aligned}
& Q=m_{e}\left(\frac{1}{24} a^{2}+\frac{1}{24} c^{2}+r_{g}^{2}\right)+m_{i}\left(r_{i}^{2}+2 r_{g}^{2}\right), \\
& J=b, \\
& X=\frac{\left(R+R_{e}\right)}{L}, \\
& Y=-\frac{K_{m}}{L}, \\
& O\left(z_{1}\right)=\left(\frac{\pi B^{2} r_{i}^{2} r_{g}^{2} \sin z_{1}}{\mu\left[h^{2}+2 r_{g}^{2}\left(1-\cos z_{1}\right)\right]^{0.5}}+\frac{\pi B^{2} r_{i}^{2} r_{g}^{2} \cos z_{1}}{\mu\left[h^{2}+2 r_{g}^{2}\left(1+\sin z_{1}\right)\right]^{0.5}}-\frac{\pi B^{2} r_{i}^{2} r_{g}^{2} \sin z_{1}}{\mu\left[h^{2}+2 r_{g}^{2}\left(1+\cos z_{1}\right)\right]^{0.5}}-\frac{\pi B^{2} r_{i}^{2} r_{g}^{2} \cos z_{1}}{\mu\left[h^{2}+2 r_{g}^{2}\left(1-\sin z_{1}\right)\right]^{0.5}}\right. \\
& +\frac{\pi B^{2} r_{i}^{2} r_{g}^{2} \sin \left(z_{1}-(\pi / 4)\right)}{\mu\left[h^{2}+2 r_{g}^{2}\left(1-\cos \left(z_{1}-(\pi / 4)\right)\right)\right]^{0.5}}+\frac{\pi B^{2} r_{i}^{2} r_{g}^{2} \cos \left(z_{1}-(\pi / 4)\right)}{\mu\left[h^{2}+2 r_{g}^{2}\left(1+\sin \left(z_{1}-(\pi / 4)\right)\right)\right]^{0.5}}-\frac{\pi B^{2} r_{i}^{2} r_{g}^{2} \sin \left(z_{1}-(\pi / 4)\right)}{\mu\left[h^{2}+2 r_{g}^{2}\left(1+\cos \left(z_{1}-(\pi / 4)\right)\right)\right]^{0.5}} \\
& \left.-\frac{\pi B^{2} r_{i}^{2} r_{g}^{2} \cos \left(z_{1}-(\pi / 4)\right)}{\mu\left[h^{2}+2 r_{g}^{2}\left(1-\sin \left(z_{1}-(\pi / 4)\right)\right)\right]^{0.5}}\right) \text {. }
\end{aligned}
$$


The generator nonlinearities of electricity generators are symmetric as $n_{r}=n_{l}$ in equation (4); consequently, we can express the fictitious input $v$ of equation (4) as

$$
v=\left\{\begin{array}{ll}
n_{l} u, & u \geq w_{r}, \\
n_{l} u, & w_{l}<u<w_{r}, \\
n_{l} u, & u \leq w_{l},
\end{array}+ \begin{cases}-n_{l} w_{r}, & u \geq w_{r}, \\
-n_{l} u, & w_{l}<u<w_{r} . \\
-n_{l} w_{l}, & u \leq w_{l},\end{cases}\right.
$$

After some mathematical operations, the fictitious input $v$ of equation (7) is expressed as

$$
\begin{aligned}
v & =n_{l} u-P(u), \\
P(u) & = \begin{cases}n_{l} w_{r}, & u \geq w_{r}, \\
n_{l} u, & w_{l}<u<w_{r}, \\
n_{l} w_{l}, & u \leq w_{l} .\end{cases}
\end{aligned}
$$

We notice that the generator nonlinearities $P(u)$ are bounded as

$$
|P(u)| \leq \bar{P}
$$

Remark 1. Even the mathematical model for the electricity generators of equations (3) and (4) seems to be simple, the complexity in this mathematical model is mainly focused on the magnets interaction nonlinearities $O\left(z_{1}\right) \in \mathfrak{R}$, such that the design and validation of this mathematical model resulted in two publications $[1,2]$.

Remark 2. From equations (5) and (6), it is observed that the magnet interaction nonlinearities $O\left(z_{1}\right) \in \mathfrak{R}$ are the most complex part in the mathematical model of equations (3) and (4), while $Q, J, X$, and $Y$ are scalar constants corresponding to the electricity generators. Thus, these magnet interaction nonlinearities $O\left(z_{1}\right) \in \mathfrak{R}$ are mainly focused on this research.

Now, we express the proportional derivation and sliding mode regulators due to they will be utilized for the comparisons in a future section. [3-5]

We express the proportional derivation regulator as

$$
u=\frac{1}{n_{l}}\left\{-K_{1} \widetilde{z}_{1}-K_{2} \widetilde{z}_{2}\right\} .
$$

We express the sliding mode regulator as [6-8]

$$
\begin{aligned}
& u=\frac{1}{n_{l}}\left\{-K_{1} \widetilde{z}_{1}-K_{2} \widetilde{z}_{2}-K \operatorname{sign}\left(\widetilde{z}_{2}\right)\right\}, \\
& \operatorname{sign}\left(\widetilde{z}_{2}\right)= \begin{cases}1, & \widetilde{z}_{2}>0, \\
0, & \widetilde{z}_{2}=0, \\
-1, & \widetilde{z}_{2}<0 .\end{cases}
\end{aligned}
$$

\section{Sliding Mode Regulator with Sine Mapping}

In this section, we address the design and stabilization of the sliding mode regulator with sine mapping.
We notice that the magnet interaction nonlinearities $O\left(z_{1}\right)$ of equation (3) are bounded as

$$
\left|O\left(z_{1}\right)\right| \leq \bar{O}
$$

Since we use the reference speed states as $z_{2}^{d}=0$, we consider the stabilization case, and the $z_{1}^{d} \in \mathfrak{R}$ reference position is a constant in this research. We express the sliding mode regulator with sine mapping $u$ as

$$
\begin{aligned}
u & =\frac{1}{n_{l}}\left\{-\cos \left(\widetilde{z}_{1}\right)^{T} K_{1} \sin \left(\widetilde{z}_{1}\right)-K_{2} \widetilde{z}_{2}-K \operatorname{sat}\left(\widetilde{z}_{2}\right)\right\}, \\
\operatorname{sat}\left(\widetilde{z}_{2}\right) & = \begin{cases}1, & \widetilde{z}_{2}>1, \\
\widetilde{z}_{2}, & \left|\widetilde{z}_{2}\right| \leq 1, \\
-1, & \widetilde{z}_{2}<-1,\end{cases}
\end{aligned}
$$

where $K$ is a constant such as $\bar{O}+\bar{P} \leq K$, where $\bar{O}$ is in equation (12) and $\bar{P}$ is in equation (9). It is relevant to note that we do not know the behaviors of $O\left(z_{1}\right)$ and $P(u)$, and we use their upper bounds $\bar{O}$ and $\bar{P}$.

Remark 3. Since we consider the stabilization case, we do not require the application of the sine mapping to the term $K_{2} \widetilde{z}_{2}$, i.e., since $z_{2}^{d}=0$ and $\widetilde{z}_{2}=z_{2}$, we obtain small values in $\widetilde{z}_{2}$. Since the state $z_{3}$ is dependent of the other states $z_{1}, z_{2}$, the stabilization of the states $z_{1}, z_{2}$ yields the stabilization of the state $z_{3}$ and we do not require the state $z_{3}$ in the regulator.

Remark 4. The convergence speed of the suggested regulator can be affected by choosing the gains $K_{1}, K_{2}$, and $K$. The complexity in the implementation of the suggested regulator is in the programming of the saturation mapping sat $(\cdot)$; nevertheless, this complexity also is in all the regulators which use mappings.

In Figure 2, we show the sliding mode regulator with sine mapping of equations (13), (12), and (9) termed SMWS for the stabilization in the electricity generators of equations (3) and (4) termed EGM.

From Figure 3, it is shown that the proportional derivation regulator of equation (10) and [3-5] termed $\mathrm{PD}$, the sliding model regulator of equations (11), (12,) and (9) and [6-8] termed SM, and the suggested sliding mode regulator with sine mapping of equations (13), (12), and (9) termed SMWS use the proportional gain $K_{1}$ and the derivation gain $K_{2}$. Furthermore, the existence, similarities, and differences between PD, SM, and SMWS are detailed as follows: the sliding model regulator starts from the proportional derivation regulator with one additional term of the signum mapping used to reach the stabilization, the sliding mode regulator with sine mapping starts from the sliding mode regulator with the modifications that the saturation mapping is used to reach a smoother performance instead of the signum mapping, and the sine mapping is applied to reach an upper bound of the proportional gain error.

Now, we will discuss the stabilization of the regulator. 


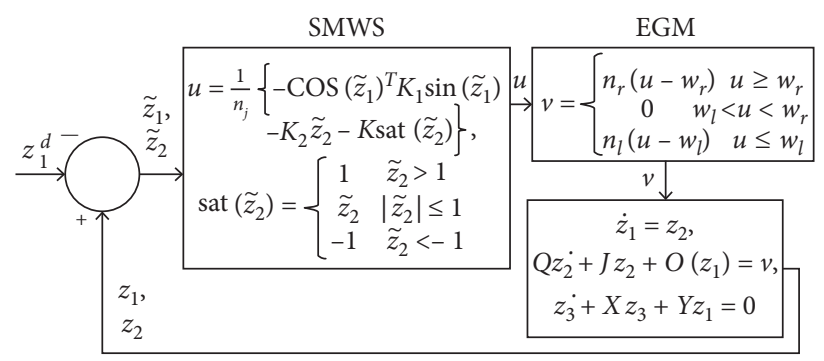

FIGURE 2: Sliding mode regulator with sine mapping.

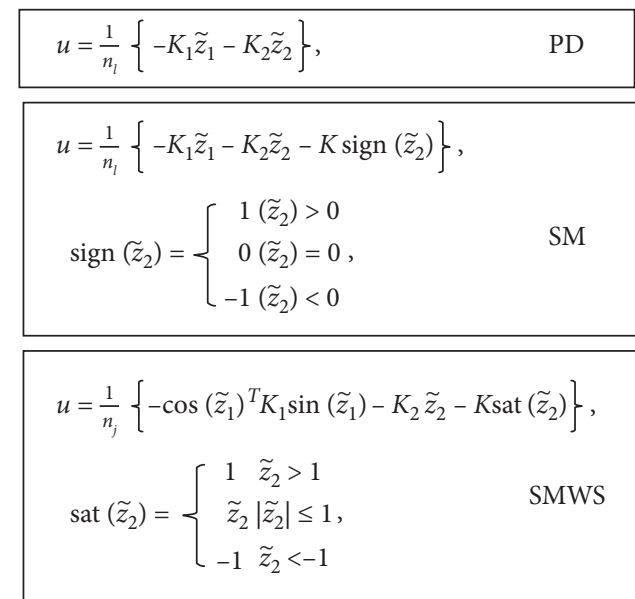

Figure 3: Relation between PD, SM, and SMWS.

Theorem 1. The stabilization in the regulator error of the sliding mode regulator with sine mapping of equations (13), (12), and (9), and electricity generators of equations (3) and (4) are ensured, and the speed regulator error $\widetilde{z}_{2}$ will converge to

$$
\limsup _{T \longrightarrow \infty}\left\|\widetilde{z}_{2}\right\|^{2}=0,
$$

with $T$ as the final time, $\widetilde{z}_{2}=z_{2}$, the magnets interaction nonlinearities and generator nonlinearities are bounded as $\left|O\left(z_{1}\right)\right| \leq \bar{O},|P(u)| \leq \bar{P}$, and $\bar{O}+\bar{P} \leq K$.
Proof. We denote the candidate mapping as

$$
V_{1}=\frac{1}{2} \widetilde{z}_{2}^{T} Q \widetilde{z}_{2}+\frac{1}{2} \sin \left(\widetilde{z}_{1}\right)^{T} K_{1} \sin \left(\widetilde{z}_{1}\right)
$$

with $Q$ as the positive constant of equation (3) and $K_{1}$ as the positive constant of equation (13). We denote $\widetilde{z}_{2}=z_{2}$, we substitute equations (13) and (8) into equation (3), and we obtain the closed-loop model as

$$
\begin{aligned}
Q \dot{z}_{2}+J z_{2}+O\left(z_{1}\right)=v & =n_{l} u-P(u) \\
& =n_{l} \frac{1}{n_{l}}\left\{-\cos \left(\widetilde{z}_{1}\right)^{T} K_{1} \sin \left(\widetilde{z}_{1}\right)-K_{2} \widetilde{z}_{2}-K \operatorname{sat}\left(\widetilde{z}_{2}\right)\right\}-P(u) \\
& \Rightarrow Q \dot{\widetilde{z}}_{2}=-\cos \left(\widetilde{z}_{1}\right)^{T} K_{1} \sin \left(\widetilde{z}_{1}\right)-K_{2} \widetilde{z}_{2}-J \widetilde{z}_{2}-O\left(z_{1}\right)-P(u)-K \operatorname{sat}\left(\widetilde{z}_{2}\right)
\end{aligned}
$$


We use the fact $\widetilde{z}_{2}=z_{2}$, and we obtain the derivation of equation (15) as

$$
\dot{V}_{1}=\widetilde{z}_{2}^{T} Q \dot{\widetilde{z}}_{2}+\widetilde{z}_{2}^{T} \cos \left(\widetilde{z}_{1}\right)^{T} K_{1} \sin \left(\widetilde{z}_{1}\right),
$$

with $\dot{\widetilde{z}}_{1}=\dot{z}_{1}-\dot{z}_{1}^{d}=z_{2}-z_{2}^{d}=z_{2}=\widetilde{z}_{2}$ and $\dot{\widetilde{z}}_{2}=\dot{z}_{2}$. We substitute last equation (16) into equation (17) as

$$
\begin{aligned}
\dot{V}_{1} & =\widetilde{z}_{2}^{T} Q \dot{\bar{z}}_{2}+\widetilde{z}_{2}^{T} \cos \left(\widetilde{z}_{1}\right)^{T} K_{1} \sin \left(\widetilde{z}_{1}\right), \\
& \Rightarrow \dot{V}_{1}=\widetilde{z}_{2}^{T}\left\{-\cos \left(\widetilde{z}_{1}\right)^{T} K_{1} \sin \left(\widetilde{z}_{1}\right)-K_{2} \widetilde{z}_{2}-J \widetilde{z}_{2}-O\left(z_{1}\right)-P(u)-K \operatorname{sat}\left(\widetilde{z}_{2}\right)\right\} \widetilde{z}_{2}^{T} \cos \left(\widetilde{z}_{1}\right)^{T} K_{1} \sin \left(\widetilde{z}_{1}\right), \\
& \Rightarrow \dot{V}_{1}=-\widetilde{z}_{2}^{T} K_{2} z_{2}-\widetilde{z}_{2}^{T} J \widetilde{z}_{2}-\widetilde{z}_{2}^{T} O\left(z_{1}\right)-\widetilde{z}_{2}^{T} P(u)-\widetilde{z}_{2}^{T} K \operatorname{sat}\left(\widetilde{z}_{2}\right)-\widetilde{z}_{2}^{T} \cos \left(\widetilde{z}_{1}\right)^{T} K_{1} \sin \left(\widetilde{z}_{1}\right)+\widetilde{z}_{2}^{T} \cos \left(\widetilde{z}_{1}\right)^{T} K_{1} \sin \left(\widetilde{z}_{1}\right) .
\end{aligned}
$$
is as

After some mathematical operations, $\dot{V}_{1}$ of equation (18)

$\dot{V}_{1}=-\widetilde{z}_{2}^{T} K_{2} \widetilde{z}_{2}-\widetilde{z}_{2}^{T} J \widetilde{z}_{2}-\widetilde{z}_{2}^{T} O\left(z_{1}\right)-\widetilde{z}_{2}^{T} P(u)-\widetilde{z}_{2}^{T} K \operatorname{sat}\left(\widetilde{z}_{2}\right)$.

Equation (19) can be expressed as

$$
\dot{V}_{1}=-\widetilde{z}_{2}^{T}\left[K_{2}+J\right] \widetilde{z}_{2}-\widetilde{z}_{2}^{T} O\left(z_{1}\right)-\widetilde{z}_{2}^{T} P(u)-\widetilde{z}_{2}^{T} K \operatorname{sat}\left(\widetilde{z}_{2}\right) .
$$

\section{Form} equation $O\left(z_{1}\right) \leq\left|O\left(z_{1}\right)\right|+|P(u)| \leq \bar{O}+\bar{P} \leq K$, and from equation (13), $\operatorname{sat}\left(\widetilde{z}_{2}\right)=\left\{\begin{array}{ll}1 & \widetilde{z}_{2}>1 \\ \widetilde{z}_{2} & \left|\widetilde{z}_{2}\right| \leq 1 \\ -1 & \widetilde{z}_{2}<-1\end{array}\right.$, we notice that there are three cases of the saturation mapping: (1) if $\widetilde{z}_{2}>1$, then $\operatorname{sat}\left(\widetilde{z}_{2}\right)=1$ and $\widetilde{z}_{2}=\left|\widetilde{z}_{2}\right|$, and we substitute into equation (20) as

$$
\begin{aligned}
\dot{V}_{1} & \leq-\widetilde{z}_{2}^{T}\left[K_{2}+J\right] \widetilde{z}_{2}+\left|\widetilde{z}_{2}\right|^{T} \bar{O}+\left|\widetilde{z}_{2}\right|^{T} \bar{P}-\left|\widetilde{z}_{2}\right|^{T} K, \\
& \Rightarrow \dot{V}_{1} \leq-\widetilde{z}_{2}^{T}\left[K_{2}+J\right] \widetilde{z}_{2} .
\end{aligned}
$$

(2) If $\left|\widetilde{z}_{2}\right| \leq 1$, then sat $\left(\widetilde{z}_{2}\right)=\widetilde{z}_{2}$ and $\widetilde{z}_{2}^{T} \widetilde{z}_{2}=\left|\widetilde{z}_{2}\right|^{T}\left|\widetilde{z}_{2}\right|$, and we substitute into equation (20) as

$$
\begin{aligned}
\dot{V}_{1} & =-\widetilde{z}_{2}^{T}\left[K_{2}+J\right] \widetilde{z}_{2}+\left|\widetilde{z}_{2}\right|^{T} \bar{O}+\left|\widetilde{z}_{2}\right|^{T} \bar{P}-\widetilde{z}_{2}^{T} \widetilde{z}_{2} K, \\
& \Rightarrow \dot{V}_{1}=-\widetilde{z}_{2}^{T}\left[K_{2}+J\right] \widetilde{z}_{2}+\left|\widetilde{z}_{2}\right|^{T} \bar{O}+\left|\widetilde{z}_{2}\right|^{T} \bar{P}-\left|\widetilde{z}_{2}\right|^{T}\left|\widetilde{z}_{2}\right| K, \\
& \Rightarrow \dot{V}_{1}=-\widetilde{z}_{2}^{T}\left[K_{2}+J\right] \widetilde{z}_{2}-\left|\widetilde{z}_{2}\right|^{T}\left[\left|\widetilde{z}_{2}\right| K-\bar{O}-\bar{P}\right], \\
& \Rightarrow \dot{V}_{1}=-\widetilde{z}_{2}^{T}\left[K_{2}+J\right] \widetilde{z}_{2},
\end{aligned}
$$

since, in this case, $\left|\widetilde{z}_{2}\right| \leq 1$, $\left|\widetilde{z}_{2}\right| K-\bar{O}-\bar{P} \geq 0 \Rightarrow \bar{O}+\bar{P} \leq\left|\widetilde{z}_{2}\right| K \leq K$. (3) If $\widetilde{z}_{2}<-1$, then sat $\left(\widetilde{z}_{2}\right)=-1$ and $\widetilde{z}_{2}=-\left|\widetilde{z}_{2}\right|$, and we substitute into equation (20) as

$$
\begin{aligned}
\dot{V}_{1} & =-\widetilde{z}_{2}^{T}\left[K_{2}+J\right] \widetilde{z}_{2}-\left(-\left|\widetilde{z}_{2}\right|^{T}\right) O\left(z_{1}\right)-\left(-\left|\widetilde{z}_{2}\right|^{T}\right) P(u)-\left(-\left|\widetilde{z}_{2}\right|^{T}\right) K(-1), \\
& \Rightarrow \dot{V}_{1} \leq-\widetilde{z}_{2}^{T}\left[K_{2}+J\right] \widetilde{z}_{2}+\left|\widetilde{z}_{2}\right|^{T} \bar{O}+\left|\widetilde{z}_{2}\right|^{T} \bar{P}-\left|\widetilde{z}_{2}\right|^{T} K \\
& \Rightarrow \dot{V}_{1} \leq-\widetilde{z}_{2}^{T} K_{2} \widetilde{z}_{2} .
\end{aligned}
$$

From equations (21)-(23), the three cases have the same inequality expressed as

$$
\dot{V}_{1} \leq-\widetilde{z}_{2}^{T}\left[K_{2}+J\right] \widetilde{z}_{2} .
$$

From the results of $[5,6]$, the stabilization of the regulator error is ensured. We integrate equation (24) from the initial time 0 to the final time $T$ as

$$
\begin{aligned}
& \int_{0}^{T} \widetilde{z}_{2}^{T}\left[K_{2}+J\right] \widetilde{z}_{2} \mathrm{~d} t \leq V_{1,0}-V_{1, T} \leq V_{1,0}, \\
& \Rightarrow \frac{\left[K_{2}+J\right]}{T} \int_{0}^{T}\left\|\tilde{z}_{2}\right\|^{2} \mathrm{~d} t \leq \frac{1}{T} \int_{0}^{T} \widetilde{z}_{2}^{T}\left[K_{2}+J\right] \tilde{z}_{2} \mathrm{~d} t \leq \frac{1}{T} V_{1,0},
\end{aligned}
$$

and we apply the limsup to both sides of the last inequality of equation (25) as $T \longrightarrow \infty$

$$
\underset{T \longrightarrow \infty}{\limsup }\left(\frac{1}{T} \int_{0}^{T}\left\|\widetilde{z}_{2}\right\|^{2} \mathrm{~d} t\right) \leq \frac{V_{1,0}}{\left[K_{2}+J\right]}\left[\limsup _{T \longrightarrow \infty}\left(\frac{1}{T}\right)\right]=0 .
$$

If $T \longrightarrow \infty$, then $\left\|\tilde{z}_{2}\right\|^{2}=0$, and we comply with equation (14).

Remark 5. From equations (1) and (2), it can be seen that the angle speed behavior affects the angle position and current behaviors in the electricity generators. Then, the angle speed convergence of equation (14) is used to reach some chosen 
constant behaviors for the angle position and current in the electricity generators.

Remark 6. This research focused on electricity generators. Nevertheless, our suggested regulator could be applied to other plants with similar structure to equations (3) and (4), such as are the motors, machines, robots, pendulums, or cranes.

Remark 7. There are several stabilization results such as the exponential stabilization $[14,17,18,20]$, the asymptotic stabilization $[5,6,16,22,23]$, the uniformly ultimately boundedness [9-11], the feedback stabilization [15, 24], the stochastic stabilization $[12,21]$, or the mean square stabilization [13]. This research focused on the asymptotic stabilization.

\section{Simulations}

In this section, we compare the sliding mode regulator with sine mapping of equations (13), (12), and (9) termed SMWS, the proportional derivation regulator of equations (10), (12), and (9), and [3-5] termed PD, and the sliding mode regulator of equations (11), (12), and (9), and [6-8] termed SM for the stabilization of the two electricity generators of equations (3) and (4). The existence, similarities, and differences between PD, SM, and SMWS are detailed in Figure 3. Our goal in the regulators is that the angle position, angle speed, and current in electricity generators must reach some chosen constant references for the angle position, angle speed, and current as fast as possible in presence of the magnet interaction nonlinearities and generator nonlinearities. The chosen constant references in the angle position are higher or equal to zero, while the chosen constant references in the angle speed and current are equal to zero. We utilize the root mean square error (RMSE) for the comparisons as

$$
\operatorname{RMSE}=\left(\frac{1}{T} \int_{0}^{T} \widetilde{z}^{2} \mathrm{~d} t\right)^{1 / 2}
$$

with $\widetilde{z}^{2}=\widetilde{z}_{1}^{2}+\widetilde{z}_{2}^{2}+\widetilde{z}_{3}^{2}$ for the states error or $\tilde{z}^{2}=v^{2}$ for the fictitious input error.

Electricity generator with two static magnets and two dynamic magnets.

We express the electricity generator with two static magnets and two dynamic magnets in equations (1)-(5).

PD of [3-5] is expressed by equations (10), (12), and (9), with parameters $K_{1}=100$ and $K_{2}=0.1$.

SM of [6-8] is expressed by equations (11), (12), and (9), with parameters $K_{1}=100, K_{2}=0.1$, and $K=0.5$.

SMWS is expressed by equations (13), (12), and (9), with parameters $K_{1}=100, K_{2}=0.1$, and $K=0.5$.

We compare the fictitious input in Figure 4 and a zoom in Figure 5, we compare the states in Figure 6 and a zoom in Figure 7, and we show the RMSE of equation (27) in Figure 6

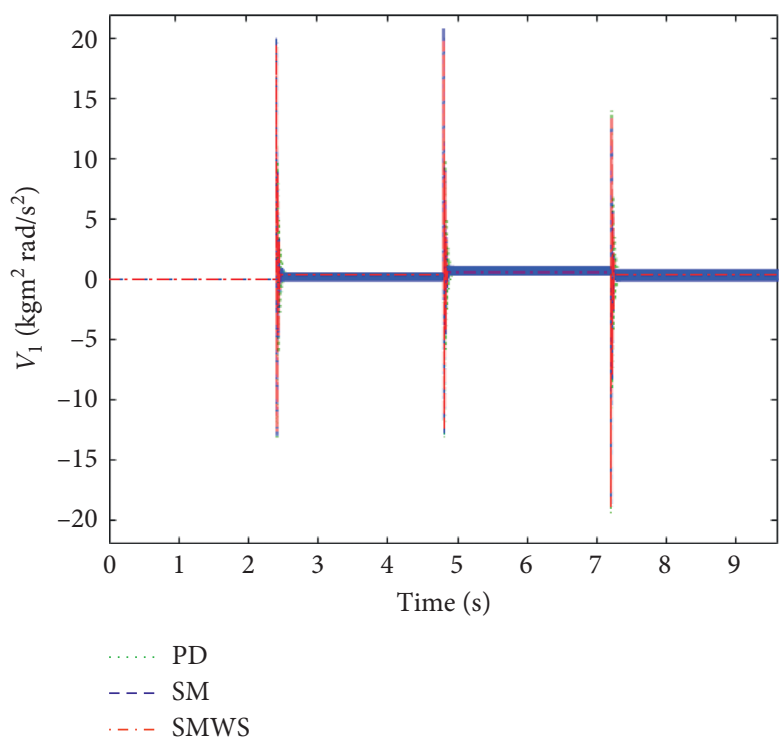

FIGURE 4: Fictitious input of the first electricity generator.

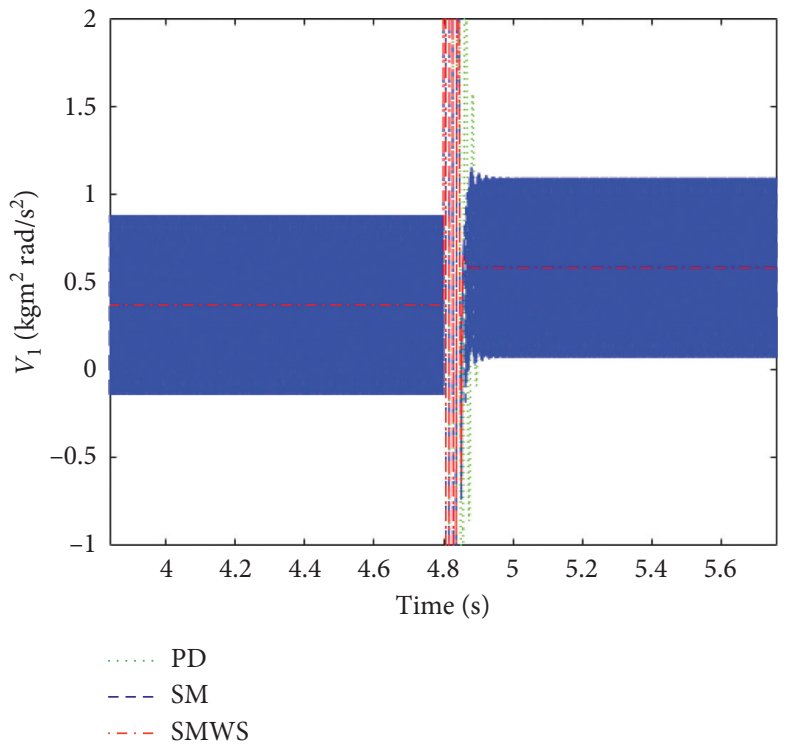

FIgURe 5: A zoom in the fictitious input of the first electricity generator.

and Table 5 for the electricity generator with two static magnets and two dynamic magnets.

In Figures 6 and 7, since the angle position, angle speed, and current of SMWS reach better the constant references in the angle position, angle speed, and current than the angle position, angle speed, and current of PD and SM, we can see that SMWS has the best performance. In Figures 4 and 5, the fictitious input for SMWS is smaller than for PD and SM. And in Figure 8 and Table 5, since the RMSE for the SMWS is smaller than for PD and SM, we can show that SMWS has the best performance. 

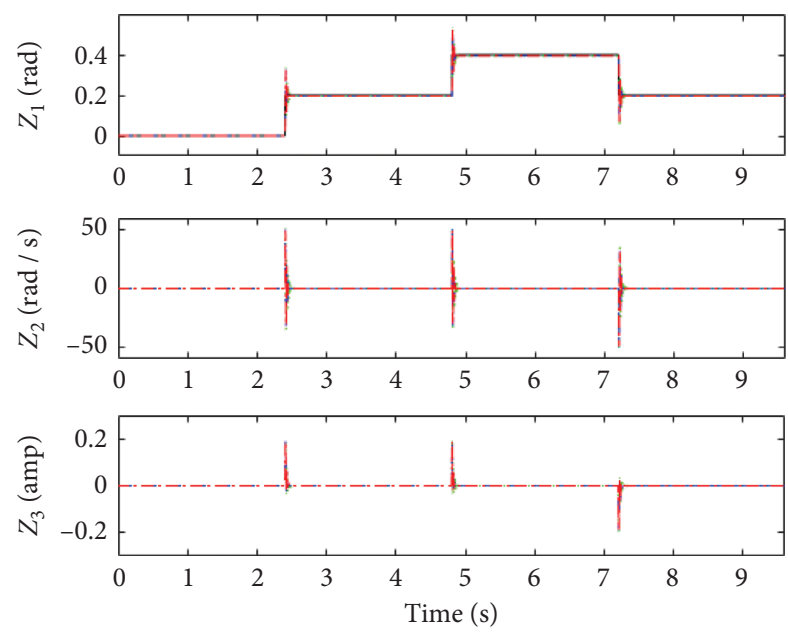

- Reference

PD

-- - SM

-.. SMWS

Figure 6: States of the first electricity generator.
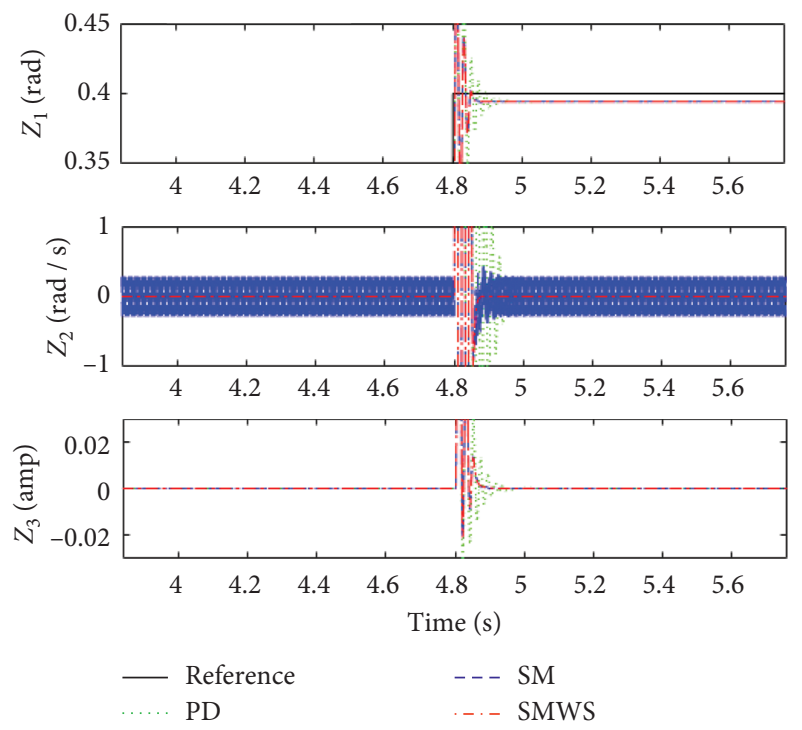

FIgURE 7: A zoom in the states of the first electricity generator.

TABLE 5: Comparisons for the first electricity generator.

\begin{tabular}{lcc}
\hline & RMSE for $\tilde{z}$ & RMSE for $v$ \\
\hline PD & 2.0154 & 0.7386 \\
SM & 1.8226 & 0.7463 \\
SMWS & 1.7835 & 0.6656 \\
\hline
\end{tabular}

Electricity generator with four static magnets and four dynamic magnets.

We express the electricity generator with four static magnets and four dynamic magnets in equations (2)-(6).

PD of [3-5] is expressed by equations (10), (12), and (9), with parameters $K_{1}=100$ and $K_{2}=0.1$.
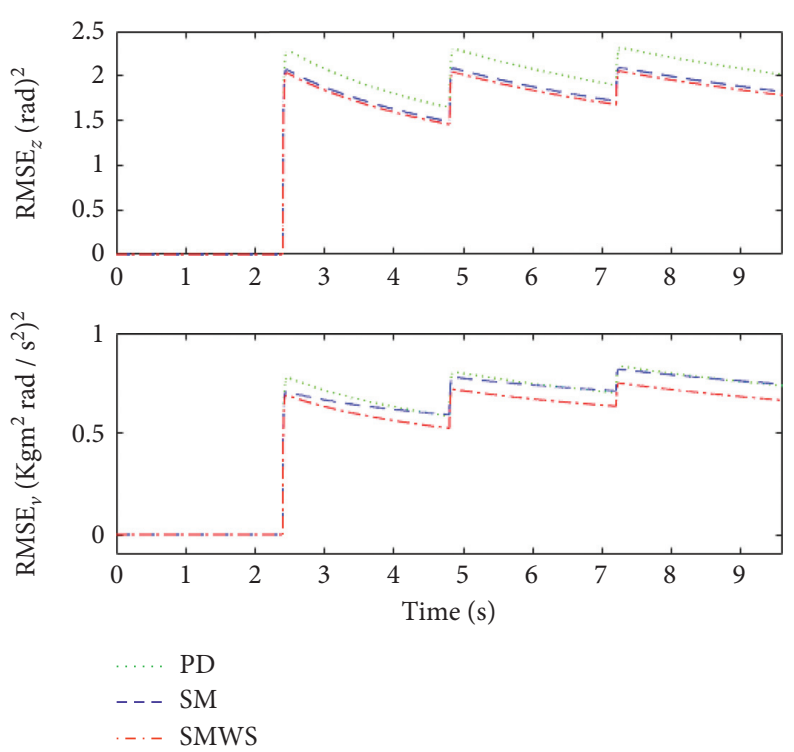

FIGURE 8: RMSE of the first electricity generator.

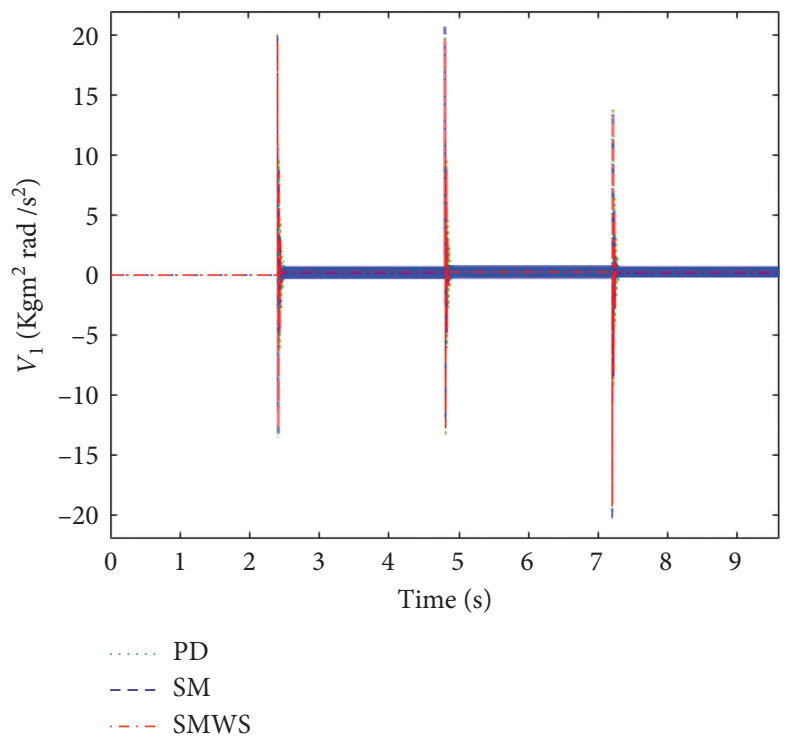

FIgURE 9: Fictitious input of the second electricity generator.

SM of [6-8] is expressed by equations (11), (12), and (9), with parameters $K_{1}=100, K_{2}=0.1$, and $K=0.5$. SMWS is expressed by equations (13), (12), and (9), with parameters $K_{1}=100, K_{2}=0.1$, and $K=0.5$.

We compare the fictitious inputs in Figure 9 and a zoom in Figure 10, we compare the states in Figure 11 and a zoom in Figure 12, and we show the RMSE of equation (27) in Figure 13 and Table 6 for the electricity generator with four static magnets and four dynamic magnets.

In Figures 11 and 12, since the angle position, angle speed, and current of SMWS reach better the constant references in the angle position, angle speed, and current than the angle position, angle speed, and current of PD and 


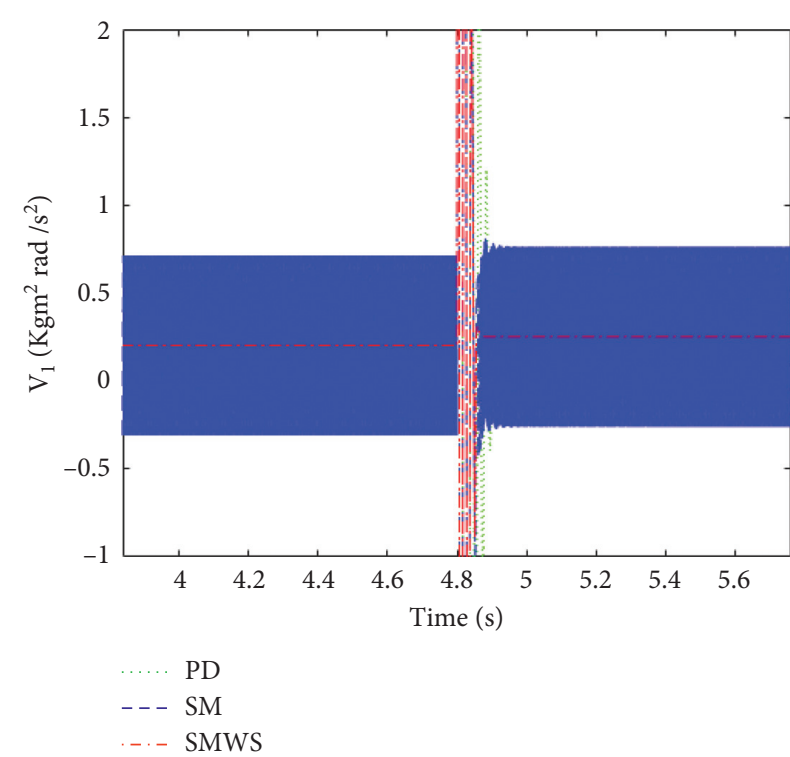

FIgURE 10: A zoom in the fictitious input of the second electricity generator.
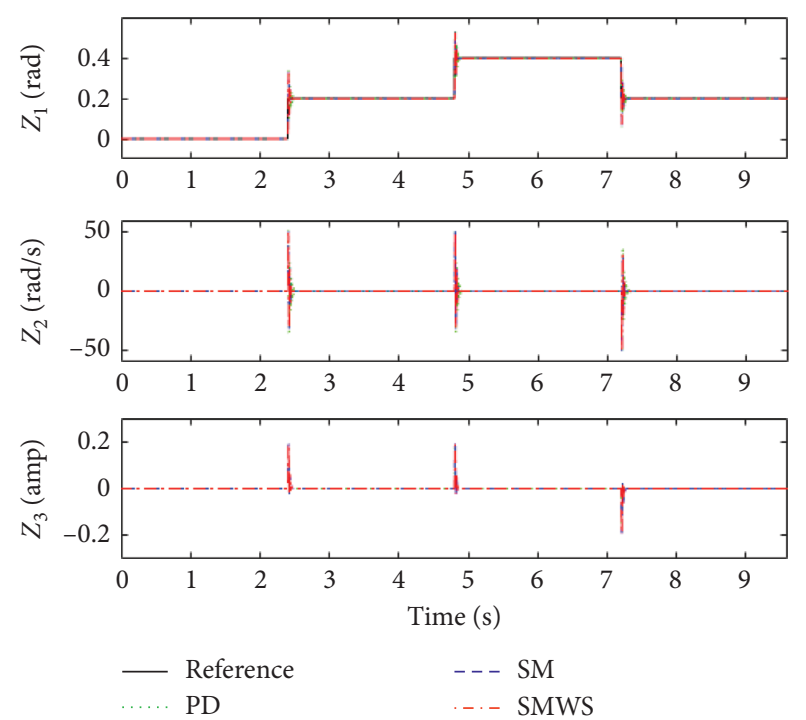

FIgURE 11: States of the second electricity generator.

SM, we can see that SMWS has the best performance. In Figures 9 and 10, the fictitious input for SMWS is smaller than for PD and SM. And in Figure 13 and Table 6, since the RMSE for the SMWS is smaller than for PD and SM, we can show that SMWS has the best performance.

Remark 8. The course of RMSE is not monotonically increasing in Figures 8 and 13 even that it is indirectly suggested in equation (27) based on the following two reasons: (a) the final time $T$ should increase for more simulation time, which is the denominator of equation (27), and (b) our goal is that the state error $\widetilde{z}^{2}=\widetilde{z}_{1}^{2}+\widetilde{z}_{2}^{2}+\widetilde{z}_{3}^{2}$ or the fictitious input
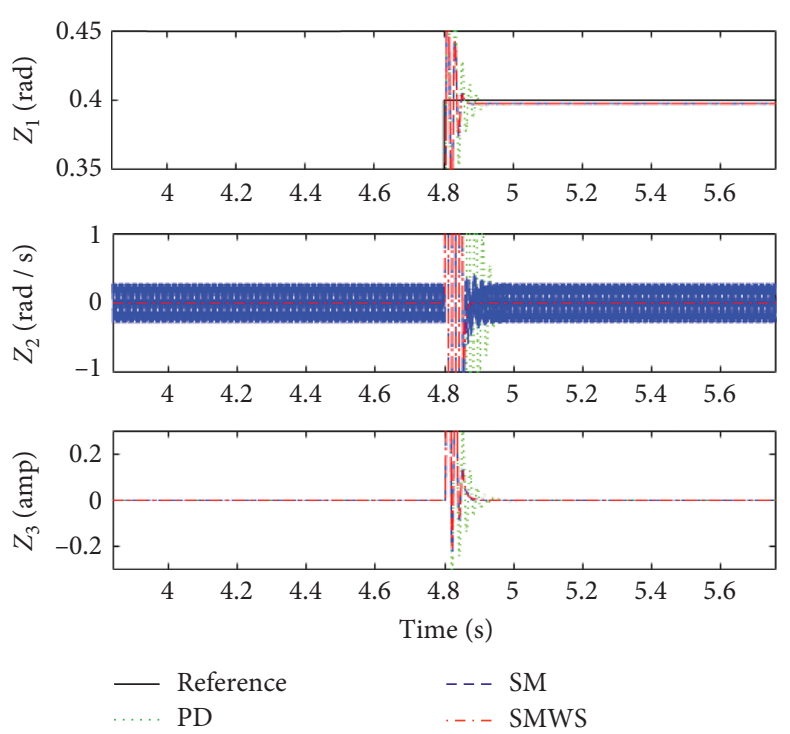

Figure 12: A zoom in the states of the second electricity generator.
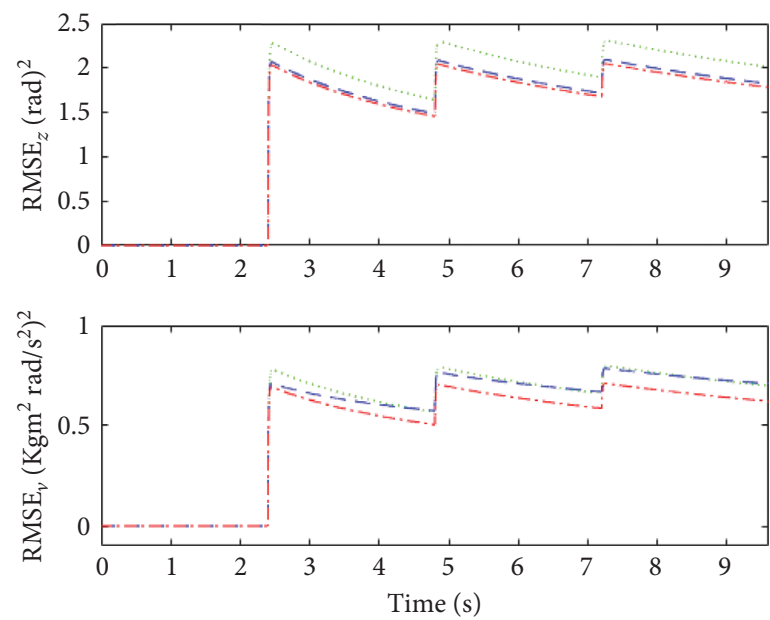

PD

-.- SM

...- SMWS

FIGURE 13: RMSE of the second electricity generator.

TABLE 6: Comparisons for the second electricity generator.

\begin{tabular}{lcc}
\hline & RMSE for $\tilde{z}$ & RMSE for $v$ \\
\hline PD & 2.0108 & 0.6995 \\
SM & 1.8230 & 0.7098 \\
SMWS & 1.7821 & 0.6224 \\
\hline
\end{tabular}

error $\tilde{z}^{2}=v^{2}$ should decrease for more simulation time, which is the numerator of equation (27). The mentioned reasons result in the decreasing of RMSE. 
Remark 9. Since Figures 4, 5, 9, and 10 describe the impulses used as the excitation to start moving these electricity generators and Figures 6, 7, 11, and 12 describe the angle position, angle speed, and current used as the responses of these electricity generators, the simulations could represent the real experiments.

\section{Conclusions}

In this research, we suggest a sliding mode regulator with sine mapping for the stabilization of two electricity generators being affected by unknown nonlinearities. In the simulations, we show that since angle position, angle speed, and current of our sliding mode regulator with sine mapping reach better the constant references of angle position, angle speed, and current than the angle position, angle speed, and current of the proportional derivation and sliding mode regulators, we can see that suggested regulator has the best performance in presence of the magnets interaction nonlinearities and generator nonlinearities. Our regulator illustrates the viability, efficiency, and potential of the approach especially relevant in electricity generators. Our suggested technique could also be applied to other mechatronic systems such as the electricity motors, machines, robots, pendulums, or cranes. The drawback of our regulator is that it is designed for models with structure of electricity generators, for models with other structures, the design should require modifications. In the future, we will apply our suggested regulator for the stabilization of robots or pendulums being affected by unknown nonlinearities.

\section{Appendix}

In this section, we express the tables used in this research.

\section{Data Availability}

The simulation data used to support this research are available from the second author upon request to the email rubio.josedejesus@gmail.com.

\section{Conflicts of Interest}

The authors declare that there are no conflicts of interest regarding the publication of this paper.

\section{Acknowledgments}

This research was financed by the Instituto Politécnico Nacional, Secretaría de Investigación y Posgrado, Comisión de Operación y Fomento de Actividades Académicas, and Consejo Nacional de Ciencia y Tecnología.

\section{References}

[1] J. J. Rubio, A. Aguilar, J. A. Meda-Campaña, G. Ochoa, R. Balcazar, and J. Lopez, "An electricity generator based on the interaction of static and dynamic magnets," IEEE Transactions on Magnetics, vol. 55, no. 8, Article ID 8204511, 2019.
[2] A. Aguilar, J. D. J. Rubio, J. A. Meda-Campaña et al., "Movable and immovable magnets for two machines," International Journal of Applied Electromagnetics and Mechanics, vol. 63, no. 2, pp. 229-248, 2020.

[3] J. O. Escobedo-Alva, E. C. Garcia-Estrada, L. A. ParamoCarranza, J. A. Meda-Campaña, and R. Tapia-Herrera, "Theoretical application of a hybrid observer on altitude tracking of quadrotor losing GPS signal," IEEE Access, vol. 6, pp. 76900-76908, 2018.

[4] J. A. Meda-Campaña, "On the estimation and control of nonlinear systems with parametric uncertainties and noisy outputs," IEEE Access, vol. 6, pp. 31968-31973, 2018.

[5] J. D. J. Rubio, Z. Zamudio, J. Pacheco, and D. Mújica Vargas, "Proportional derivative control with inverse dead-zone for pendulum systems," Mathematical Problems in Engineering, vol. 2013, Article ID 173051, 9 pages, 2013.

[6] J. J. Rubio, "Sliding mode control of robotic arms with deadzone," IET Control, Theory and Applications, vol. 11, no. 8, pp. 1214-1221, 2017.

[7] C. Aguilar-Ibanez, H. Sira-Ramirez, M. S. Suarez-Castanon, and R. Garrido, "Robust trajectory-tracking control of a pvtol under crosswind," Asian Journal of Control, vol. 21, pp. 1293-1306, 2019.

[8] C. Aguilar-Ibanez and M. S. Suarez-Castanon, "A trajectory planning based controller to regulate an uncertain 3D overhead crane system," International Journal of Applied Mathematics and Computer Science, vol. 29, no. 4, pp. 693702, 2019.

[9] Y. Chang, Y. Wang, F. E. Alsaadi, and G. Zong, "Adaptive fuzzy output-feedback tracking control for switched stochastic pure-feedback nonlinear systems," International Journal of Adaptive Control and Signal Processing, vol. 33, no. 10, pp. 1567-1582, 2019.

[10] Y. Chang, S. Zhang, N. D. Alotaibi, and A. F. Alkhateeb, "Observer-based adaptive finite-time tracking control for a class of switched nonlinear systems with unmodeled dynamics," IEEE Access, vol. 8, 2020.

[11] Y. Wang, Y. Chang, A. F. Alkhateeb, and N. D. Alotaibi, "Adaptive fuzzy output-feedback tracking control for switched nonstrict-feedback nonlinear systems with prescribed performance," Circuits, Systems, and Signal Processing, 2020.

[12] B. Jiang and H. R. Karimi, "Further criterion for stochastic stability analysis of semi-Markovian jump linear systems," International Journal of Robust and Nonlinear Control, vol. 30, no. 7, pp. 2689-2700, 2020.

[13] B. Wang and Q. Zhu, "Stability analysis of discrete time semimarkov jump linear systems," IEEE Transactions on Automatic Control, vol. 65, no. 12, pp. 5415-5421, 2020.

[14] W. Hu, Q. Zhu, and H. R. Karimi, "Some improved razumikhin stability criteria for impulsive stochastic delay differential systems," IEEE Transactions on Automatic Control, vol. 64, no. 12, pp. 5207-5213, 2019.

[15] H. Wang and Q. Zhu, "Global stabilization of a class of stochastic nonlinear time-delay systems with SISS inverse dynamics," IEEE Transactions on Automatic Control, vol. 65, no. 10, pp. 4448-4455, 2020.

[16] C. Yuan, S. Song, Q. Gao, H. R. Karimi, L. Pekar, and S. Guo, "A novel frequency-domain approach for the exact range of imaginary spectra and the stability analysis of LTI systems with two delays," IEEE Access, vol. 8, pp. 36595-36601, 2020.

[17] Q. Zhu, "Stability analysis of stochastic delay differential equations with Lévy noise," Systems \& Control Letters, vol. 118, pp. 62-68, 2018. 
[18] Q. Zhu and T. Huang, "Stability analysis for a class of stochastic delay nonlinear systems driven by G-Brownian motion," Systems \& Control Letters, vol. 140, Article ID 104699, 2020.

[19] M. Li, H. Chen, and X. Li, "Synchronization analysis of complex dynamical networks subject to delayed impulsive disturbances," Complexity, vol. 2020, Article ID 5285046, 12 pages, 2020.

[20] Q. Zhu, "Stabilization of stochastic nonlinear delay systems with exogenous disturbances and the event-triggered feedback control," IEEE Transactions on Automatic Control, vol. 64, no. 9, pp. 3764-3771, 2019.

[21] A. Azizi, "A case study on computer-based analysis of the stochastic stability of mechanical structures driven by white and colored noise: utilizing artificial intelligence techniques to design an effective active suspension system," Complexity, vol. 2020, Article ID 9321928, 17 pages, 2020.

[22] W. Ji, J. Qiu, and H. R. Karimi, "Fuzzy-model-based output feedback sliding-mode control for discrete-time uncertain nonlinear systems," IEEE Transactions on Fuzzy Systems, vol. 28, no. 8, pp. 1519-1530, 2020.

[23] B. Xiao, X. Yang, H. R. Karimi, and J. Qiu, "Asymptotic tracking control for a more representative class of uncertain nonlinear systems with mismatched uncertainties," IEEE Transactions on Industrial Electronics, vol. 66, no. 12, pp. 9417-9427, 2019.

[24] Q. Zhu and H. Wang, "Output feedback stabilization of stochastic feedforward systems with unknown control coefficients and unknown output function," Automatica, vol. 87, pp. 166-175, 2018. 